\title{
ON SOME FUNCTIONAL EQUATIONS ON STANDARD OPERATOR ALGEBRAS
}

\author{
Irena Kosi-UlbL AND Joso VUKMAN \\ University of Maribor, Slovenia
}

\begin{abstract}
The main purpose of this paper is to prove the following result. Let $X$ be a real or complex Banach space, let $L(X)$ be the algebra of all bounded linear operators on $X$, let $A(X) \subseteq L(X)$ be a standard operator algebra, and let $T: A(X) \rightarrow L(X)$ be an additive mapping satisfying the relation $T\left(A^{2 n+1}\right)=\sum_{i=1}^{2 n+1}(-1)^{i+1} A^{i-1} T(A) A^{2 n+1-i}$, for all $A \in A(X)$ and some fixed integer $n \geq 1$. In this case $T$ is of the form $T(A)=A B+B A$, for all $A \in A(X)$ and some fixed $B \in L(X)$. In particular, $T$ is continuous.
\end{abstract}

Throughout, $R$ will represent an associative ring. Given an integer $n>1$, a ring $R$ is said to be $n$-torsion free, if for $x \in R, n x=0$ implies $x=0$. An additive mapping $x \mapsto x^{*}$ on a ring $R$ is called an involution if $(x y)^{*}=y^{*} x^{*}$ and $x^{* *}=x$ hold for all pairs $x, y \in R$. A ring equipped with an involution is called a ring with involution or ${ }^{*}$-ring. Recall that a ring $R$ is prime if for $a, b \in R, a R b=(0)$ implies that either $a=0$ or $b=0$, and is semiprime in case $a R a=(0)$ implies $a=0$. Let $A$ be an algebra over the real or complex field and let $B$ be a subalgebra of $A$. A linear mapping $D: B \rightarrow A$ is called a linear derivation in case $D(x y)=D(x) y+x D(y)$ holds for all pairs $x, y \in B$. In case we have a ring $R$ an additive mapping $D: R \rightarrow R$ is called a derivation if $D(x y)=D(x) y+x D(y)$ holds for all pairs $x, y \in R$ and is called a Jordan derivation in case $D\left(x^{2}\right)=D(x) x+x D(x)$ is fulfilled for all $x \in R$. A derivation $D$ is inner in case there exists $a \in R$, such that $D(x)=a x-x a$ holds for all $x \in R$. Every derivation is a Jordan derivation. The converse is in general not true. A classical result of Herstein [7] asserts that any

2000 Mathematics Subject Classification. 46K15, 39B05.

Key words and phrases. Prime ring, semiprime ring, Banach space, standard operator algebra.

This research has been supported by the Research Council of Slovenia. 
Jordan derivation on a 2-torsion free prime ring is a derivation. Cusack [4] generalized Herstein's result to 2-torsion free semiprime rings. It should be mentioned that Beidar, Brešar, Chebotar and Martindale have considerably generalized Herstein's theorem (see [2, Theorem 4.4]. For explanation of the symmetric Martindale ring of quotients of a semiprime ring $R$, which will be denoted by $Q_{S}(R)$, we refer to [1]. Let $X$ be a real or complex Banach space, and let $L(X)$ and $F(X)$ denote the algebra of all bounded linear operators on $X$, and the ideal of all finite rank operators in $L(X)$, respectively. An algebra $A(X) \subseteq L(X)$ is said to be standard in case $F(X) \subseteq A(X)$. Let us point out that any standard algebra is prime, which is a consequence of Hahn-Banach theorem. A projection $P \in L(H)$, where $H$ is a complex Banach space, is called bicircular in case all mappings of the form $e^{i \alpha} P+e^{i \beta}(I-P)$, where $I$ denotes the identity operator, are isometric for all pairs of real numbers $\alpha, \beta$.

Vukman, Kosi-Ulbl and Eremita [14] have proved the following result.

TheOREM 1 ([14, Theorem 2.1]). Let $R$ be a 2-torsion free semiprime ring. Suppose that $T: R \rightarrow R$ is an additive mapping satisfying the relation

$$
T(x y x)=T(x) y x-x T(y) x+x y T(x)
$$

for all pairs $x, y \in R$. In this case $T$ is of the form

$$
2 T(x)=q x+x q,
$$

for all $x \in R$ and some fixed $q \in Q_{s}(R)$.

Putting in the relation (1) $y=x$ we obtain

$$
T\left(x^{3}\right)=T(x) x^{2}-x T(x) x+x^{2} T(x), \quad x \in R .
$$

Fošner and Vukman [6] have recently proved the following result.

TheOREM 2 ([6, Theorem 3.2]). Let $R$ be a 2-torsion free prime ring. Suppose that $T: R \rightarrow R$ is an additive mapping satisfying the relation (2) for all $x \in R$. In this case $T$ is of the form

$$
4 T(x)=q x+x q
$$

for all $x \in R$ and some fixed $q \in Q_{s}(R)$.

From the relation (2) one obtains by induction the following generalization

$$
T\left(x^{2 n+1}\right)=\sum_{i=1}^{2 n+1}(-1)^{i+1} x^{i-1} T(x) x^{2 n+1-i}, \quad x \in R,
$$

where $n \geq 1$ is some fixed integer. In this paper we consider the relation (3) in standard operator algebras. 
Theorem 3. Let $X$ be a real or complex Banach space and let $A(X)$ be a standard operator algebra on $X$. Suppose $T: A(X) \rightarrow L(X)$ is an additive mapping satisfying the relation

$$
T\left(A^{2 n+1}\right)=\sum_{i=1}^{2 n+1}(-1)^{i+1} A^{i-1} T(A) A^{2 n+1-i},
$$

for all $A \in A(X)$ and some fixed integer $n \geq 1$. In this case $T$ is of the form

$$
T(A)=A B+B A,
$$

for all $A \in A(X)$ and some fixed $B \in L(X)$. In particular, $T$ is continuous.

Let us point out that in the theorem above we obtain as a result the continuity of $T$ under purely algebraic assumptions concerning the mapping $T$. Therefore, the above result might be of some interest from the automatic continuity point of view. In the proof of Theorem 3 we shall use Theorem 2 .

Proof of TheOREM 3. We have the relation

$$
T\left(A^{2 n+1}\right)=\sum_{i=1}^{2 n+1}(-1)^{i+1} A^{i-1} T(A) A^{2 n+1-i}, \quad A \in A(X) .
$$

Let $A$ be from $F(X)$ and let $P \in F(X)$ be a projection with $A P=P A=$ $A$. Putting $A+m P(m \in \mathbb{N})$ for $A$ in the relation (4) and comparing the coefficients of $m^{2 n}$, we obtain

$$
\begin{aligned}
(2 n+1) T & (A) \\
= & (T(A) P+2 n T(P) A)+(P T(A)+2 n A T(P)) \\
& +(-P T(A) P+P T(A) P-\cdots+P T(A) P-P T(A) P) \\
& +(-1+2-\cdots+(2 n-2)-(2 n-1))(A T(P) P+P T(P) A) .
\end{aligned}
$$

The above equation reduces to

$$
\begin{aligned}
(2 n+1) T(A)= & T(A) P+P T(A)-P T(A) P \\
& +n(2 T(P) A+2 A T(P)-A T(P) P-P T(P) A) .
\end{aligned}
$$

Multiplying the above relation from both sides by $P$ we obtain

$$
2 P T(A) P=A T(P) P+P T(P) A,
$$

which reduces (5) to

$$
\begin{aligned}
(2 n+1) T(A)= & T(A) P+P T(A)-P T(A) P \\
& +2 n(T(P) A+A T(P)-P T(A) P) .
\end{aligned}
$$

Left multiplication of the above relation by $P$ gives

$$
P T(A)=P T(P) A+A T(P)-P T(A) P .
$$

Similarly, we obtain

$$
T(A) P=A T(P) P+T(P) A-P T(A) P .
$$


Adding up (8) and (9), and applying (6), we obtain

$$
T(A) P+P T(A)=A T(P)+T(P) A .
$$

Inserting this in (7), we get

$$
T(A)=T(P) A+A T(P)-P T(A) P .
$$

From (10) and (6) we obtain

$$
2 T(A)=A(2 T(P)-T(P) P)+(2 T(P)-P T(P)) A .
$$

Hence $2 T(A)=A Q+R A$ with $Q=2 T(P)-T(P) P$ and $R=2 T(P)-P T(P)$; note that $A Q A=A R A$. Direct calculation yields

$$
T\left(A^{3}\right)=T(A) A^{2}-A T(A) A+A^{2} T(A) .
$$

From the relation (10) one can conclude that $T$ maps $F(X)$ into itself. Therefore we have an additive mapping $T: F(X) \rightarrow F(X)$ satisfying the relation (11) for all $A \in F(X)$. Since $F(X)$ is prime one can apply Theorem 2, which means that $T$ is of the form

$$
4 T(A)=A C+C A,
$$

for all $A \in F(X)$ and some $C \in Q_{s}(F(X))$. Since $Q_{s}(F(X))=L(X)$ (this is the direct consequence of [1, Theorem 4.3.8] and [8, p.78, Example 5]), one can conclude that $T$ is of the form

$$
T(A)=A B+B A,
$$

for all $A \in F(X)$ and some $B \in L(X)$. It remains to prove that the relation (12) holds on $A(X)$ as well. Let us introduce $T_{1}: A(X) \rightarrow L(X)$ by $T_{1}(A)=$ $A B+B A$ and consider $T_{0}=T-T_{1}$. The mapping $T_{0}$ is, obviously, linear and satisfies the relation (4). Besides, $T_{0}$ vanishes on $F(X)$. It is our aim to prove that $T_{0}$ vanishes on $A(X)$ as well. Let $A \in A(X)$, let $P$ be an one-dimensional projection and

$$
S=A+P A P-(A P+P A)
$$

We have $T_{0}(S)=T_{0}(A)$ and $S P=P S=0$. We have

$$
T_{0}\left(A^{2 n+1}\right)=\sum_{i=1}^{2 n+1}(-1)^{i+1} A^{i-1} T_{0}(A) A^{2 n+1-i},
$$


for all $A \in A(X)$. Applying the above relation we obtain

$$
\begin{aligned}
\sum_{i=1}^{2 n+1}( & -1)^{i+1} S^{i-1} T_{0}(S) S^{2 n+1-i} \\
= & T_{0}\left(S^{2 n+1}\right)=T_{0}\left(S^{2 n+1}+P\right)=T_{0}\left((S+P)^{2 n+1}\right) \\
= & \sum_{i=1}^{2 n+1}(-1)^{i+1}(S+P)^{i-1} T_{0}(S+P)(S+P)^{2 n+1-i} \\
= & T_{0}(A)\left(S^{2 n}+P\right)+\sum_{i=2}^{2 n}(-1)^{i+1}\left(S^{i-1}+P\right) T_{0}(A)\left(S^{2 n+1-i}+P\right) \\
& +\left(S^{2 n}+P\right) T_{0}(A) \\
= & T_{0}(A) S^{2 n}+T_{0}(A) P+\sum_{i=2}^{2 n}(-1)^{i+1} S^{i-1} T_{0}(A) S^{2 n+1-i} \\
& +\sum_{i=2}^{2 n}(-1)^{i+1} P T_{0}(A) S^{2 n+1-i}+\sum_{i=2}^{2 n}(-1)^{i+1} S^{i-1} T_{0}(A) P \\
& +S^{2 n} T_{0}(A)+P T_{0}(A)-P T_{0}(A) P \\
= & \sum_{i=1}^{2 n+1}(-1)^{i+1} S^{i-1} T_{0}(A) S^{2 n+1-i}+\sum_{i=2}^{2 n}(-1)^{i+1} P T_{0}(A) S^{2 n+1-i} \\
+ & \sum_{i=2}^{2 n}(-1)^{i+1} S^{i-1} T_{0}(A) P+T_{0}(A) P+P T_{0}(A)-P T_{0}(A) P .
\end{aligned}
$$

We have therefore

$$
\begin{aligned}
& \sum_{i=2}^{2 n}(-1)^{i+1} P T_{0}(A) S^{2 n+1-i}+\sum_{i=2}^{2 n}(-1)^{i+1} S^{i-1} T_{0}(A) P \\
& +T_{0}(A) P+P T_{0}(A)-P T_{0}(A) P=0 .
\end{aligned}
$$

Multiplying the above relation from both sides by $P$ we obtain

$$
P T_{0}(A) P=0,
$$

which reduces the relation (13) to

$$
\sum_{i=2}^{2 n}(-1)^{i+1} P T_{0}(A) S^{2 n+1-i}+\sum_{i=2}^{2 n}(-1)^{i+1} S^{i-1} T_{0}(A) P+T_{0}(A) P+P T_{0}(A)=0 .
$$

Right multiplication of the above relation by $P$ gives

$$
\sum_{i=1}^{2 n}(-1)^{i+1} S^{i-1} T_{0}(A) P=0 .
$$


Putting in the above relation $-A$ for $A$ (note that in this case $S$ becomes $-S$ ), and comparing the relation so obtained with the relation (14), we obtain

$$
\sum_{i=1}^{n} S^{2(i-1)} T_{0}(A) P=0 .
$$

Inserting $m A(m \in \mathbb{N})$ instead of $A$ we get (since $S$ is then replaced by $m S$ )

$$
\sum_{i=1}^{n} S^{2(i-1)} T_{0}(A) P m^{2 i-1}=0 .
$$

The coefficient of $m$ is equal to $T_{0}(A) P$. We have therefore $T_{0}(A) P=0$. Since $P$ is an arbitrary one-dimensional projection, it follows that $T_{0}(A)=0$, for any $A \in A(X)$, which completes the proof of the theorem.

In [9] one can find the following result.

Theorem 4 ([9, Theorem 1$])$. Let $X$ be a real or complex Banach space and let $A(X)$ be a standard operator algebra on $X$. Suppose $D: A(X) \rightarrow L(X)$ is a linear mapping satisfying the relation

$$
D\left(A^{n}\right)=\sum_{i=1}^{n} A^{i-1} D(A) A^{n-i},
$$

for all $A \in A(X)$ and some integer $n>1$. In this case $D$ is of the form

$$
D(A)=A B-B A,
$$

for all $A \in A(X)$ and some $B \in L(X)$, which means that $D$ is a linear derivation. In particular, $D$ is continuous.

The history of the above result goes back to the classical result of Chernoff $[3]$ (see also $[12,13,16])$ which states that in case there exists a linear derivation $D$, which maps a standard operator algebra $A(X)$ into $L(X)$, where $X$ is a real or complex Banach space, then $D$ is of the form $D(A)=A B-B A$, for all $A \in A(X)$ and some $B \in L(X)$. Theorem 4 generalizes the result we have just mentioned above. Let us point out that in Theorem 3 we assumed that $T$ is an additive mapping, while in Theorem 4 we have stronger assumption that $D$ is linear. In general Chernoff's result and therefore also Theorem 4 cannot be proved by assuming that $D$ is additive as shown by Šmrl in [12].

In the proof of our next result we apply Theorem 3 and Theorem 4.

Corollary 5. Let $X$ be a real or complex Banach space and let $A(X)$ be a standard operator algebra on $X$. Suppose $D, G: A(X) \rightarrow L(X)$ are linear 
mappings satisfying the relations

$$
\begin{aligned}
D\left(A^{2 n+1}\right)= & D(A) A^{2 n}+A G(A) A^{2 n-1}+A^{2} D(A) A^{2 n-2}+\cdots \\
& +A^{2 n-1} G(A) A+A^{2 n} D(A) \\
G\left(A^{2 n+1}\right)= & G(A) A^{2 n}+A D(A) A^{2 n-1}+A^{2} G(A) A^{2 n-2}+\cdots \\
& +A^{2 n-1} D(A) A+A^{2 n} G(A)
\end{aligned}
$$

for all $A \in A(X)$ and some fixed integer $n \geq 1$. In this case $D$ and $G$ are of the form

$$
D(A)=A B-C A, \quad G(A)=A C-B A,
$$

for all $A \in A(X)$ and some $B$ and $C$ from $L(X)$. In particular, $D$ and $G$ are continuous.

Proof. Adding up (16) with (17) we obtain

$$
F\left(A^{2 n+1}\right)=\sum_{i=1}^{2 n+1} A^{i-1} F(A) A^{2 n+1-i},
$$

for all $A \in A(X)$, where $F$ stands for $D+G$. Subtracting (17) from (16) we obtain

$$
H\left(A^{2 n+1}\right)=\sum_{i=1}^{2 n+1}(-1)^{i+1} A^{i-1} H(A) A^{2 n+1-i},
$$

for all $A \in A(X)$, where $H$ denotes $D-G$. Now, applying Theorem 3 and Theorem 4 , we obtain

$$
F(A)=D(A)+G(A)=A B-B A,
$$

and

$$
H(A)=D(A)-G(A)=A C+C A,
$$

for all $A \in A(X)$ and some fixed $B$ and $C$ from $L(X)$. From (20) and (21) we obtain $2 D(A)=A(B+C)+(C-B) A, 2 G(A)=A(B-C)-(B+C) A$. Replacing $\frac{1}{2}(B+C)$ by $B$ and $\frac{1}{2}(B-C)$ by $C$ we obtain $D(A)=A B-C A$, $G(A)=A C-B A$ for all $A \in A(X)$, which completes the proof of the corollary.

Stachó and Zalar $([10,11])$ investigated bicircular projections on $C^{*}$ algebra $L(H)$, the algebra of all bounded linear operators on a complex Hilbert space $H$. According to Proposition 3.4 in [10] every bicircular projection $P: L(H) \rightarrow L(H)$ satisfies the relation

$$
P(x y x)=P(x) y x-x P\left(y^{*}\right)^{*} x+x y P(x)
$$

for all pairs $x, y \in L(H)$. Fošner and Ilišević [5] investigated the above functional equation in 2 -torsion free semiprime ${ }^{*}$-rings. They expressed the solution of the equation (22) in terms of derivations and so-called double centralizers. Vukman showed that applying more direct approach makes it possible 
to prove a more general result ([15]). Fošner and Vukman ([6]) investigated the following system of functional equations on 2-torsion free prime ${ }^{*}$-rings

$$
\begin{array}{ll}
P\left(x^{3}\right)=P(x) x^{2}+x Q\left(x^{*}\right)^{*} x+x^{2} P(x), & x \in R, \\
Q\left(x^{3}\right)=Q(x) x^{2}+x P\left(x^{*}\right)^{*} x+x^{2} Q(x), & x \in R .
\end{array}
$$

The observations above lead to our next result.

Corollary 6. Let $H$ be a real or complex Hilbert space and let $A(H)$ be a standard operator algebra on $H$ which is closed under the adjoint operation. Suppose $P, Q: A(H) \rightarrow L(H)$ are linear mappings satisfying the relations

$$
\begin{aligned}
P\left(A^{2 n+1}\right)= & P(A) A^{2 n}+A Q\left(A^{*}\right)^{*} A^{2 n-1}+A^{2} P(A) A^{2 n-2}+\cdots \\
& +A^{2 n-1} Q\left(A^{*}\right)^{*} A+A^{2 n} P(A), \\
Q\left(A^{2 n+1}\right)= & Q(A) A^{2 n}+A P\left(A^{*}\right)^{*} A^{2 n-1}+A^{2} Q(A) A^{2 n-2}+\cdots \\
& +A^{2 n-1} P\left(A^{*}\right)^{*} A+A^{2 n} Q(A),
\end{aligned}
$$

for all $A \in A(H)$ and some integer $n \geq 1$. In this case $P$ and $Q$ are of the form

$$
P(A)=A B-C A, \quad Q(A)=-A B^{*}+C^{*} A,
$$

for all $A \in A(H)$ and some fixed $B, C \in L(H)$. In particular, $P$ and $Q$ are continuous.

Proof. Put $D(A)=P(A)$ and $G(A)=Q\left(A^{*}\right)^{*}$ for all $A \in A(H)$ and apply Corollary 5. There exist $B, C \in L(H)$ such that

$$
P(A)=A B-C A, \quad Q\left(A^{*}\right)^{*}=A C-B A
$$

for all $A \in A(H)$, that is

$$
P(A)=A B-C A, \quad Q(A)=-A B^{*}+C^{*} A
$$

for all $A \in A(H)$. The proof of the corollary is complete.

ACKNOWLEDGEMENTS.

The authors wish to express thanks to the referee for helpful suggestions which considerably improved the paper.

\section{REFERENCES}

[1] K. I. Beidar, W. S. Martindale III and A.V. Mikhalev, Rings with generalized identities, Marcel Dekker, Inc. New York, 1996.

[2] K. I. Beidar, M. Brešar, M.A. Chebotar and W. S. Martindale 3 rd, On Herstein's Lie map conjectures II, J. Algebra 238 (2001), 239-264.

[3] P. R. Chernoff, Representations, automorphisms, and derivations of some operator algebras, J. Functional Analysis 12 (1973), 275-289.

[4] J. Cusack, Jordan derivations on rings, Proc. Amer. Math. Soc. 53 (1975), 321-324.

[5] M. Fošner and D. Ilišević, On a class of projections on *-rings, Commun. Algebra 33 (2005), 3293-3310.

[6] M. Fošner and J. Vukman, On some equations in prime rings, Monatsh. Math. 152 (2007), 135-150. 
[7] I. N. Herstein, Jordan derivations of prime rings, Proc. Amer. Math. Soc. 8 (1957), 1104-1110.

[8] G. N. Jacobson, Structure of rings, American Mathematical Society, New York, 1956.

[9] I. Kosi-Ulbl and J. Vukman, An identity related to derivations of standard operator algebras and semisimple $H^{*}$-algebras, CUBO A Math. J., to apper.

[10] L. L. Stachó and B. Zalar, Bicircular projections on some matrix and operator spaces, Linear Algebra Appl. 384 (2004), 9-20.

[11] L. L. Stachó and B. Zalar, Bicircular projections and characterization of Hilbert spaces, Proc. Amer. Math. Soc. 132 (2004), 3019-3025.

[12] P. Šemrl, Ring derivations on standard operator algebras, J. Funct. Anal. 112 (1993), 318-324.

[13] J. Vukman, On automorphisms and derivations of operator algebras, Glasnik Mat. Ser. III 19(39) (1984), 135-138.

[14] J. Vukman, I. Kosi Ulbl and D. Eremita, On certain equations in rings, Bull. Austral. Math. Soc. 71 (2005), 53-60.

[15] J. Vukman, On functional equations related to bicircular projections, Glasnik Mat. Ser. III 41(61) (2006), 51-55.

[16] J. Vukman, On derivations of standard operator algebras and semisimple $H^{*}$-algebras, Studia Sci. Math. Hungar. 44 (2007), 57-63.

I. Kosi-Ulbl

Faculty of Mechanical Engineering

University of Maribor

Smetanova ul. 17, Maribor

Slovenia

E-mail: irena.kosi@uni-mb.si

J. Vukman

Department of Mathematics and Computer Science, FNM

University of Maribor

Koroška 160, Maribor

Slovenia

E-mail: joso.vukman@uni-mb.si

Received: 10.12.2008.

Revised: 7.1.2009. 